\title{
Filarial Parasite-Derived New Potential Bio-Therapeutic Agents For Inflammatory Bowel Diseases
}

\author{
Vishal Khatri, Nikhil Chauhan and Ramaswamy Kalyanasundaram* \\ Department of Biomedical Sciences, University of Illinois College of Medicine, Rockford, IL, USA
}

${ }^{\star}$ Corresponding author: Ramaswamy Kalyanasundaram, PhD, Department of Biomedical Sciences, University of Illinois College of Medicine, 1601 Parkview Avenue, Rockford, IL 61107, USA; Email: ramswamy@uic.edu

Received: April 19, 2019; Accepted: April 26, 2019; Published: May 01, 2019;

Inflammatory Bowel Disease (IBD) comprising of Crohn's disease (CD) and Ulcerative Colitis (UC), is a chronic and relapsing inflammatory condition of the gastrointestinal tract [1]. There has been an alarming increase in the incidence of IBD during the past decade, leading to long-term morbidity that considerably affects the quality of patient's life [1]. The incidence of UC has been rising globally since the mid-20th century [2]. Genetic factors are thought to play an important role in the pathogenesis of UC. Studies by Bengtson et al. [3] showed that the risk of developing colitis rises by 4.6 -fold when a sibling has colitis. Similarly, if one of the monozygotic twins develops colitis, the risk of the other twin developing the disease is relatively 95 times higher [4]. Dietary factors have also been reported to play a role in the development of colitis. A diet containing high amounts of refined fat, meat, and sugar are risk factors for developing colitis [5]. Unfortunately, the mainstream therapies available currently for treating colitis are largely non-specific with short-term immunosuppressive effect and nearly all of them predispose the patients to opportunistic infections and/or increased risk for cancer development [6]. The most preferred method for treating mild to moderate UC is the administration of 5-aminosalicylates (5-ASA). Patients suffering from moderate to severe UC or those who do not respond to 5-ASA can be treated with antibiotics or corticosteroids but these treatment options come with various side effects like nausea, headache, diarrhea, insomnia, osteoporosis, and non-Hodgkin lymphoma [7, 8]. Similarly, anti-TNF- $\alpha$ therapy against UC is associated with the risk of hepatosplenic T cell lymphoma [9] and the effect of anti-TNF- $\alpha$ therapy reduces with time [10]. Recent advances to study the mechanism of inflammation in UC has provided a better understanding of the underlying molecular basis of the disease, which is helping in the development of new therapeutic approaches for UC $[11,12]$.

Parasites, more specifically the helminth parasites are notorious for suppressing the inflammatory- immune responses in their host [13]. This immunosuppression or immunomodulation is a strategy used by the parasites to survive and reproduce in their host. We are beginning to unravel the complex mechanisms by which the helminth parasites are able to achieve the immunomodulation in the host, despite the vigorous immune responses generated by the host against the parasites. In general, the host-derived inflammatory responses create a hostile environment for the helminth parasite resulting in the trapping of the parasite in the tissue leading to the killing of the parasite by antibodies and inflammatory cells or seriously damaging the fecundity of the female worms so the future generations of the parasites are suppressed or prevented. Thus, the major purpose of immunosuppression by the parasite in the host appears to be to evade the host attack and escape [14]. The helminth parasites predominantly achieve this by secreting key molecules that have potent anti-inflammatory activity [15]. Even though the host inflammatory responses towards the helminth parasites are largely local, the parasite-induced immunosuppression is more generalized with a non-specific suppressive effect on other inflammatory conditions in the host [16].

It is well established that acute infection with the filarial parasites, Brugia malayi and Wuchereria bancrofti is associated with generalized immunosuppression [17]. Clearing of the parasites with chemotherapy reverses this immunosuppressive state, confirming a role for the live parasite in this generalized immunosuppression [18]. Similarly, in filarial infected subjects, immunizations with Tetanus and BCG are found to be ineffective [17]. Similar generalized immunosuppression also occurs in the host during infections with gastrointestinal nematode parasites [19]. In 1989, David Strachan [20] reported a higher incidence of allergic rhinitis and hay fever in children who have better living conditions with good water quality, hygiene, improved sanitation, and medical care. However, children from smaller families, who were exposed to several parasitic infections, do not exhibit these allergic conditions. Based on this finding, he postulated the theory of 'Hygiene Hypothesis.' Since then, several studies demonstrated the therapeutic potential of filarial parasitic infections in a variety of immune-mediated disorders such as arthritis, diabetes, multiple sclerosis, allergic and atopic conditions, and IBD [21-25]. Although controversial, several Phase 2 clinical trials show significant remission in the symptoms of IBD, MS, allergic rhinitis, autism, and peanut/tree nut allergy in patients following the treatment with live parasites [26]. Unfortunately, live parasites or ova are aesthetically not acceptable for many patients as a treatment. Another major hurdle is in obtaining regulatory approvals and product stability for live parasites because of the potential batch to batch variations. Few studies showed that worm homogenates can induce immunosuppressive activity similar to the live parasite [27]. This suggested that molecules produced by the live parasites can be used instead of the live parasites to induce the immunosuppressive effect. Since then, several laboratories started 
focusing on screening the parasite and its genome for identifying molecules that may have potential immunomodulatory activity.

Host immunomodulatory molecules have been isolated from several parasites such as Acanthocheilonema viteae, Schistosoma japonicum, Brugia malayi, Wuchereria bancrofti, Heligmosomoides polygyrus bakeri and Schistosoma mansoni $[27,28]$. Lymphatic filarial parasites are notorious for suppressing host immune responses, especially during acute infections. Few reports suggest that lymphatic filariasis infected patients often do not suffer from autoimmune diseases [29]. Therefore, there has been significant interest among researchers to identify the immunomodulatory molecules of lymphatic filarial parasites that have significant immunosuppressive effect in experimental autoimmune diseases [27, 30-34]. Several filarial derived molecules have been identified as immunomodulatory agents, such as mammalian cytokine homologs, Abundant Larval Transcript (ALT) antigens, macrophage migration inhibitory factor (MIF), cysteine proteases and venom allergen-like proteins [35-38]. This commentary will focus mainly on the biotherapeutic properties of cysteine proteases and MIF. Both these molecules possess significant anti-inflammatory property in experimental arthritis and in IBD animal models [22, 27, 32, 39-41].

Cystatins, belong to the family of cysteine protease inhibitors superfamily. The three major families of cystatins include stefins (cystatins A and B), cystatins (Cystatin C, E, and S) and kininogens [42]. The cystatins are present in almost all living organisms. They are involved in a wide array of physiological and pathological processes. Misregulation of cystatins may lead to the disease state [42]. Nematode cystatins were first described in Onchocerca volvulus [42].
Subsequently, it was shown that the parasite cystatins are involved in the regulation of the molting of the parasites, establishment of active parasitism within the hosts, modulation of cathepsin activities and suppression of inflammation, antigen presentation, and lymphocyte activation in the host $[17,42,43]$. Experiments have demonstrated that $\mathrm{Bm}$-Cystatin protease inhibitor-2 (CPI-2) blocked antigen processing of tetanus-toxoid protein in vitro by purified B cells and asparaginyl endopeptidases [42].

Although filarial and vertebrate cystatins do not share significant similarities, X-ray crystallographic studies showed conserved motifs in cystatins that form a wedge-shaped structure (Fig. 1), which blocks the active site of cysteine proteases [43]. Some of the conserved structural elements include $\mathrm{N}$-terminal signal peptide, an approximately 100 aa domain, two disulfide bonds, a central Gln-XVal-X-Gly motif and a C-terminal Pro-Trp hairpin loop [43]. Three types of cystatin proteins were identified from B. malayi. At the genomic level, the intron positions are highly conserved among the three types of $B$. malayi cystatins, which might indicate the possible adaptation to the parasitic life cycle [43]. The conserved catalytic domains of cystatins could interfere with the cysteine proteases involved in the degradation of antigens within the endosomal compartment of APC, thus preventing the presentation of peptides to MHC class II molecules [42-44]. In addition to interfering with antigen presentation, the filarial cystatins can suppress antigen-induced proliferation of human Peripheral Blood Monocytes (PBMC), and down-regulate expression of human leucocyte antigen (HLA-DR) and the costimulatory molecule CD86 on human PBMC [42,44]. These studies demonstrate potential mechanisms by which the filarial cystatins are inducing their immunomodulatory activity.

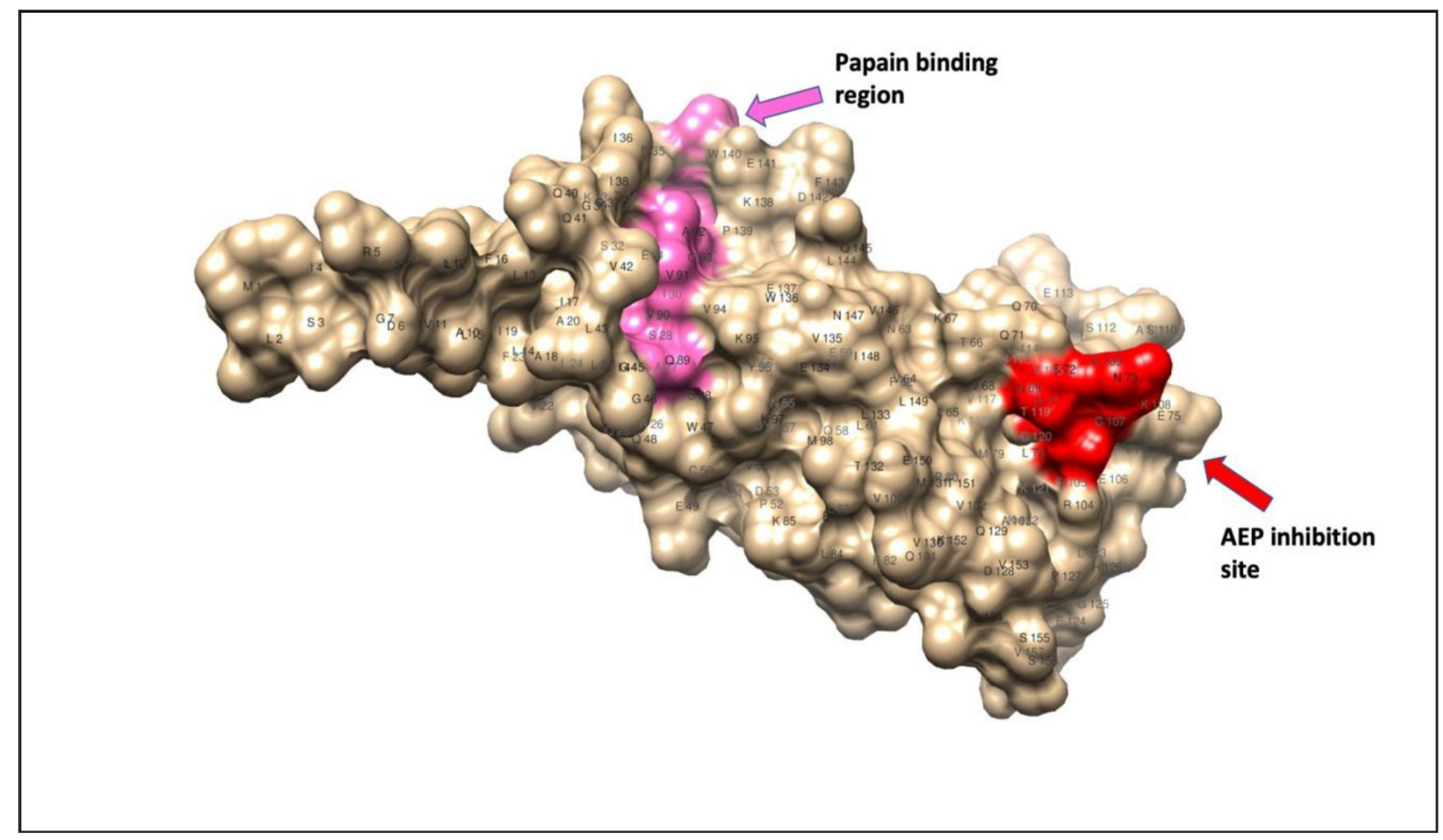

Figure 1. Homology modeling of cystatin-2 of B. malayi filarial parasite (157 a.a.) [69, 70]; 3D structure showing conserved papain-binding site (pink) and asparaginyl endopeptidase (AEP) inhibitory site (red). 
Given its potent immunoregulatory role, cystatins of helminth parasites such as Ascaris lumbricoides, Schistosoma japonicum, Acanthocheilonema viteae, Clonorchis sinensis, B. malayi and Fasciola hepatica have been extensively studied for their therapeutic potential in various inflammatory immune conditions including UC [32, 4550]. Schnoeller et al. [51] were the first one to uncover the therapeutic efficacy of a recombinant cystatin from A. vitae (Av17) in murine models of OVA-induced allergic airway responsiveness and DSSinduced colitis. Subsequent studies confirmed this immunomodulatory potential of AvCystatin for their ability to amelioration DSS-induced intestinal inflammation, characterized by decreased loss of body weight, reduction in the shortening of the colon length and minimal histopathological changes and myeloperoxidase activity in the colon tissues $[48,52]$. Cystatin from $S$. japonicum has also been widely tested against different experimental disease models [53-55]. S. japonicum recombinant cystatin ( $\mathrm{rSj}$-Cys)-treated $\mathrm{DBA} / 1$ mice were protected from CIA-induced arthritis [53]. Similarly, rSjcystatin administration has also significantly ameliorated the TNBS-induced colitis condition [54]. Likewise, recombinant cystatin from A. lumbricoides (rAl-CPI) was able to ameliorate the DSS-induced colitis in a dose-dependent manner [45]. Type I cystatin (CsStefin-1) of the liver fluke, C. sinensis has also been shown to attenuate the DSS-induced colitis [49]. Recently, the therapeutic effect of S. japonicum cystatin (Sj-Cys) was demonstrated in an experimental animal model of sepsis [55]. Similarly, administration of filarial cystatin is shown to suppress the severity of mBSA-induced arthritis in mice $[22,40]$.

Our studies using B. malayi cystatin ( $\mathrm{rBmaCys}$ ) showed that this protein could alleviate the pathology of Ulcerative Colitis (UC) in a mouse model $[32,56]$. Intraperitoneal administration of $\mathrm{rBmaCys}$ led to the reduction in the overall disease severity, decreased clinical symptoms and histopathological changes in both acute and chronic colitis [32, 56]. Although, the immunomodulatory function of $\mathrm{r} B m a$ Cys is repeatedly demonstrated, the mechanism by which the $\mathrm{rBmaCys}$ achieves this anti-inflammatory effect is not fully understood. Some of the evidence shows a role for Treg cells and IL-10 in this immunosuppressive mechanism [unpublished observations].

Another parasite-derived molecule that has potent host immunomodulatory activity is the Macrophage Migration Inhibitory Factor (MIF) (Fig. 2). This molecule is different from the mammalian homolog of the MIF. The mammalian MIF is a potent pro-inflammatory molecule and is involved in several inflammatory diseases such as psoriasis, asthma, and IBD [57]. The mammalian MIF possesses two catalytic activities a Pro-2 dependent tautomerase and a Cys-Ala-Leu-Cys (CALC) dependent thiol oxidoreductase [58]. The association between catalytic activities of MIF and its immunogenic functions is not fully studied. Mutation in Cys60Ser abolishes the proinflammatory function of mammalian MIF [59]. Peptide fragment (50aa-65aa) with redox activity is present in mammalian MIF [60]. This suggested that oxidoreductase activity is directly involved in some of the immunological functions of MIF. In contrast, Pro2 dependent tautomerase activity failed to establish a link between the catalytic activity of MIF and its glucocorticoid overriding activity [58]. The testing of these catalytic mutants should assist further dissecting the molecular basis of the catalytic activities of mammalian MIF and their immunological roles in inflammatory diseases.

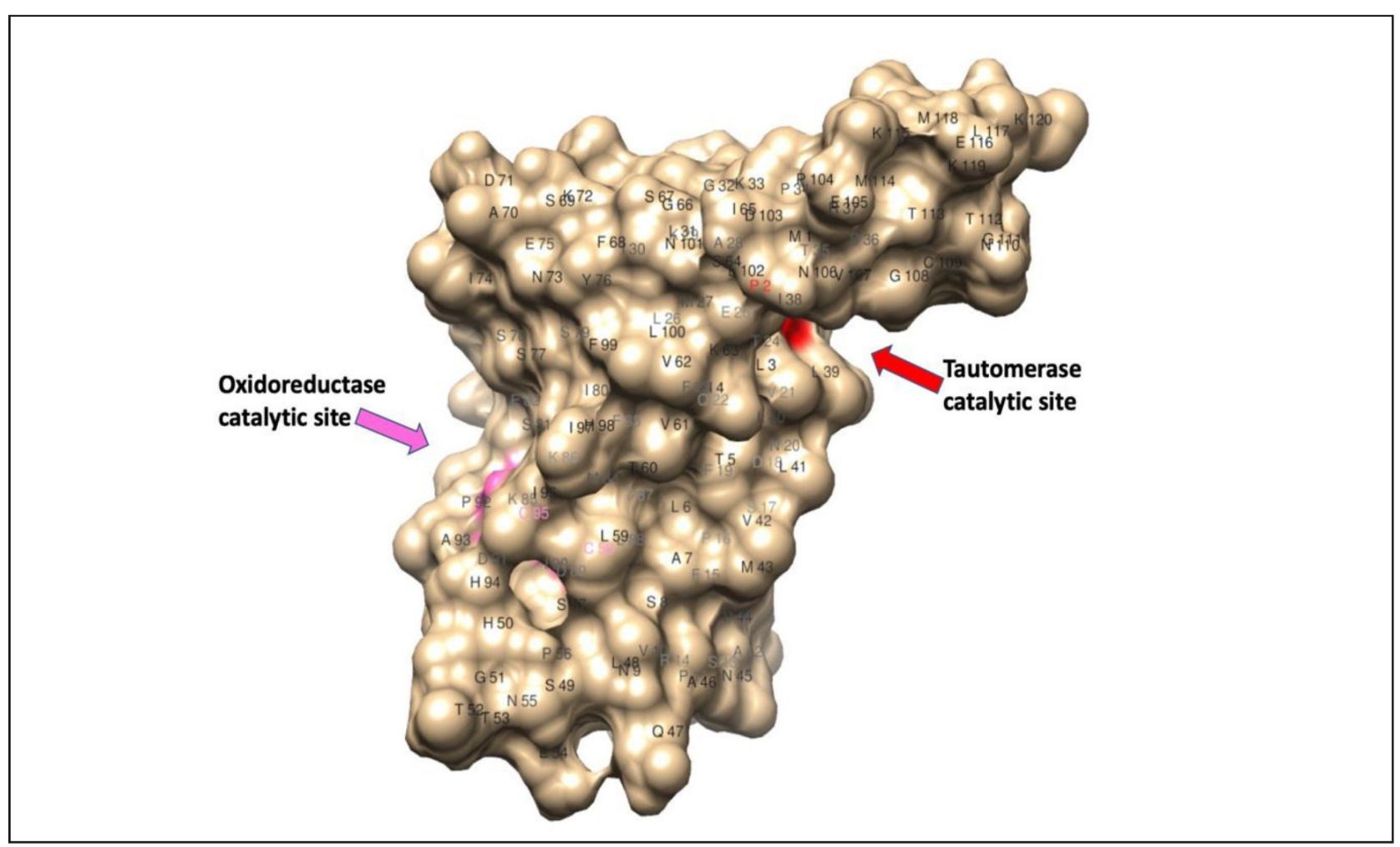

Figure 2: Homology modeling of macrophage migration inhibitory factor -2 of $W$. bancrofti filarial parasite (120 a.a.) [69, 70]; 3D structure showed the position of Pro-2mediated tautomerase catalytic site (red) and $\mathrm{C}_{58} \& \mathrm{C}_{95}$ mediated oxidoreductase catalytic site (pink). 
Two homologs of mammalian MIF, MIF-1, and MIF-2 are reported from nematode parasites with $22 \%$ and $40 \%$ similarity with mammalian MIF respectively [61]. Similar to cystatin, the parasitederived MIF can interact with CD74 receptor on antigen presenting cells and interfere with antigen presentation, suggesting that the parasite-derived MIF may be immunomodulatory [62]. B. malayi MIF-1 and MIF-2 are abundantly secreted in the excretory-secretory products of the larval stages of the parasite (microfilariae and molting L3 stages) [61, 63]. Infection with the filarial parasite results in less phagocytic and antigen processing ability in the host splenic macrophages. When soluble recombinant BmMIF-1 and BmMIF-2 were administered to mice, Ym1 expression was upregulated in the macrophages polarizing them to M2 phenotype that expressed high levels of IL-4R $[64,65]$. Similar results were obtained when macrophages were incubated in vitro with the filarial MIF. Thus, it appears that parasite-derived MIF can promote the differentiation of alternatively activated macrophages (AAM), which can regulate inflammation, promotes wound healing and tissue repair, and probably contribute to the clearance of helminth parasites [66]. These findings suggested that the helminth-derived MIF may have an immunomodulatory function. Subsequent studies showed that in a mouse model of asthma, the MIF protein of A. simplex parasite can completely prevent the accumulation of eosinophils and prevent hyperplasia of goblet cells in the lungs a resulting in the amelioration of hypersensitivity reaction in the lungs [67]. Similarly, MIF-2 protein from A. simplex parasite can also ameliorate symptoms of colitis in a DSS induced colitis mouse model [57]. This immunomodulatory effect appears to be mediated by Treg cells because MIF-2 treatment increases the Treg population in the mouse [68]. One of our recent studies showed that filarial MIF-2 also has potent immunomodulatory effect in reducing the inflammation in the colon of a mouse with DSSinduced colitis (unpublished observations). These findings suggest that both cystatin and MIF-2 from filarial parasites have potent immunomodulatory activity in reducing inflammatory changes in colitis and IBD.

In summary, IBD and other inflammatory conditions greatly hamper the normal life of the patients and there is an urgent need to develop better therapeutics. Developing biotherapeutics from helminths, more specifically filarial parasites have tremendous potential to be used as complementary and alternative medicine. Our studies identified two such molecules (cystatin and MIF-2) from the filarial parasites that have tremendous potential as small molecules for the treatment of colitis. Since the administration of these molecules significantly reduces inflammation and reverses clinical symptoms, there is significant potential for developing these molecules as biotherapeutic agents for IBD.

Keywords: Inflammatory Bowel Disease, Helminth Therapy, Cystatin, Macrophage Migration Inhibitory Factor-2, Brugia malayi, Wuchereria bancrofti, Ulcerative Colitis

\section{References}

1. Zuo T, Ng SC (2018) The Gut Microbiota in the Pathogenesis and Therapeutics of Inflammatory Bowel Disease. Front Microbiol 9: 2247. [crossref]

2. Ng SC, Shi HY, Hamidi N, Underwood FE, Tang W, Benchimol EI, et al. (2018) Worldwide incidence and prevalence of inflammatory bowel disease in the 21 st century: a systematic review of population-based studies. Lancet 390: 2769-78.
3. Bengtson MB, Solberg IC, Aamodt G, Jahnsen J, Moum B, Vatn MH, et al. (2010) Relationships between inflammatory bowel disease and perinatal factors: both maternal and paternal disease are related to preterm birth of offspring. Inflamm Bowel Dis 16: 847-55.

4. Halme L1, Paavola-Sakki P, Turunen U, Lappalainen M, Farkkila M, et al. (2006) Family and twin studies in inflammatory bowel disease. World J Gastroenterol 12: 3668-3672. [crossref]

5. Limdi JK (2018) Dietary practices and inflammatory bowel disease. Indian $J$ Gastroenterol 37: 284-292. [crossref]

6. Taghipour N, Aghdaei HA, Haghighi A, Mossafa N, Tabaei SJ, Rostami-Nejad M. (2014) Potential treatment of inflammatory bowel disease: a review of helminths therapy. Gastroenterol Hepatol Bed Bench 7: 9-16.

7. Marchioni Beery R, Kane S (2014) Current approaches to the management of newonset ulcerative colitis. Clin Exp Gastroenterol 7: 111-132. [crossref]

8. Büning C, Lochs H (2006) Conventional therapy for Crohn's disease. World J Gastroenterol 12: 4794-4806. [crossref]

9. Thai A, Prindiville T (2010) Hepatosplenic T-cell lymphoma and inflammatory bowel disease. J Crohns Colitis 4: 511-522. [crossref]

10. Monaco C, Nanchahal J, Taylor P, Feldmann M2 (2015) Anti-TNF therapy: past, present and future. Int Immunol 27: 55-62. [crossref]

11. Neurath MF (2014) New targets for mucosal healing and therapy in inflammatory bowel diseases. Mucosal Immunol 7: 6-19.

12. Cheifetz AS, Gianotti R, Luber R, Gibson PR. (2017) Complementary and Alternative Medicines Used by Patients With Inflammatory Bowel Diseases. Gastroenterology 152: 415-29.

13. Maizels RM, McSorley HJ (2016) Regulation of the host immune system by helminth parasites. J Allergy Clin Immunol 138: 666-675. [crossref]

14. Geiger A, Bossard G, Sereno D, Pissarra J, Lemesre JL, Vincendeau P, et al. (2016) Escaping Deleterious Immune Response in Their Hosts: Lessons from Trypanosomatids. Front Immunol 7: 212.

15. Adisakwattana P, Saunders SP, Nel HJ, Fallon PG (2009) Helminth-derived immunomodulatory molecules. Adv Exp Med Biol 666: 95-107. [crossref]

16. Smallwood TB, Giacomin PR, Loukas A, Mulvenna JP, Clark RJ, Miles JJ (2017) Helminth Immunomodulation in Autoimmune Disease. Front Immunol 8:453.

17. Maizels RM, Gomez-Escobar N, Gregory WF, Murray J, Zang X (2001) Immune evasion genes from filarial nematodes. Int J Parasitol 31: 889-898. [crossref]

18. Hoerauf A, Satoguina J, Saeftel M, Specht S (2005) Immunomodulation by filarial nematodes. Parasite Immunol 27: 417-429. [crossref]

19. Cooper D, Eleftherianos I (2016) Parasitic Nematode Immunomodulatory Strategies: Recent Advances and Perspectives. Pathogens 5.

20. Strachan DP (1989) Hay fever, hygiene, and household size. BMJ 299: 1259-1260. [crossref]

21. Lopes F, Matisz C, Reyes JL, Jijon H, Al-Darmaki A, Kaplan GG, et al. (2016) Helminth Regulation of Immunity: A Three-pronged Approach to Treat Colitis. Inflamm Bowel Dis 22: 2499-512.

22. Ravi Shankar Prasad Yadav VK, Nitin Amdare, Kalyan Goswami, VB Shivkumar, Nitin Gangane, Maryada Venkata Rami Reddy (2017) Evaluation of preventive effect of Brugia malayi recombinant cystatin on mBSA-induced experimental arthritis. Indian Journal of Experimental Biology 55:5.

23. Amdare N, Khatri V, Yadav RS, Tarnekar A, Goswami K, Reddy MV (2015) Brugia malayi soluble and excretory-secretory proteins attenuate development of streptozotocin-induced type 1 diabetes in mice. Parasite Immunol 37: 624-34.

24. Santiago HC, Nutman TB (2016) Human Helminths and Allergic Disease: The Hygiene Hypothesis and Beyond. Am J Trop Med Hyg 95: 746-753. [crossref]

25. Wendel-Haga M, Celius EG (2017) Is the hygiene hypothesis relevant for the risk of multiple sclerosis? Acta Neurol Scand 201: 26-30. [crossref]

26. Fleming JO, Weinstock JV (2015) Clinical trials of helminth therapy in autoimmune diseases: rationale and findings. Parasite Immunol 37: 277-92.

27. Heylen M, Ruyssers NE, Gielis EM, Vanhomwegen E, Pelckmans PA2, et al. (2014) Of worms, mice and man: an overview of experimental and clinical helminth-based therapy for inflammatory bowel disease. Pharmacol Ther 143: 153-167. [crossref]

28. Wang M, Wu L, Weng R, Zheng W, Wu Z, Lv Z (2017) Therapeutic potential of helminths in autoimmune diseases: helminth-derived immune-regulators and immune balance. Parasitol Res 116: 2065-2074.

29. Buerfent BC, Golz L, Hofmann A, Ruhl H, Stamminger W, Fricker N, et al. (2019) Transcriptome-wide analysis of filarial extract-primed human monocytes reveal changes in LPS-induced PTX3 expression levels. Sci Rep 9: 2562.

30. Adisakwattana P, Nuamtanong S, Kusolsuk T, Chairoj M, Yenchitsomanas PT, Chaisri U (2013) Non-encapsulated Trichinella spp., T. papuae, diminishes severity of DSS-induced colitis in mice. Asian Pac J Allergy Immunol 31: 106-14.

31. Broadhurst MJ, Ardeshir A, Kanwar B, Mirpuri J, Gundra UM, Leung JM, et al. (2012) Therapeutic helminth infection of macaques with idiopathic chronic diarrhea 
alters the inflammatory signature and mucosal microbiota of the colon. PLoS Pathog 8: 1003000 .

32. Khatri V, Amdare N, Tarnekar A, Goswami K, Reddy MV (2015) Brugia malayi cystatin therapeutically ameliorates dextran sulfate sodium-induced colitis in mice. J Dig Dis 16: 585-94.

33. Ferreira I, Smyth D, Gaze S, Aziz A, Giacomin P, Ruyssers N, et al. (2013) Hookworm excretory/secretory products induce interleukin-4 (IL-4)+ IL-10+ $\mathrm{CD} 4+\mathrm{T}$ cell responses and suppress pathology in a mouse model of colitis. Infect Immun 81: 2104-11.

34. Heylen M, Ruyssers NE, De Man JG, Timmermans JP, Pelckmans PA, Moreels TG, et al. (2014) Worm proteins of Schistosoma mansoni reduce the severity of experimental chronic colitis in mice by suppressing colonic proinflammatory immune responses. PLoS One 9: 110002 .

35. Chauhan N, Hoti SL (2016) Role of cysteine-58 and cysteine-95 residues in the thiol di-sulfide oxidoreductase activity of Macrophage Migration Inhibitory Factor-2 of Wuchereria bancrofti. Acta Trop 153: 14-20. [crossref]

36. Hewitson JP, Grainger JR, Maizels RM (2009) Helminth immunoregulation: the role of parasite secreted proteins in modulating host immunity. Mol Biochem Parasitol 167: 1-11. [crossref]

37. Maizels RM, Smits HH, McSorley HJ. Modulation of Host Immunity by Helminths: The Expanding Repertoire of Parasite Effector Molecules. Immunity 49: 801-18.

38. Ottesen EA (1984) Immunological aspects of lymphatic filariasis and onchocerciasis in man. Trans $R$ Soc Trop Med Hyg 78 Suppl: 9-18. [crossref]

39. Gazzinelli-Guimaraes PH, Nutman TB (2018) Helminth parasites and immune regulation. F1000Res 7. [crossref]

40. Yadav RS, Khatri V, Amdare N, Goswami K, Shivkumar VB, Gangane N, et al Immuno-Modulatory Effect and Therapeutic Potential of Brugia malayi Cystatin in Experimentally Induced Arthritis. Indian J Clin Biochem 31: 203-8.

41. Zakeri A, Hansen EP, Andersen SD, Williams AR, Nejsum P (2018) Immunomodulation by Helminths: Intracellular Pathways and Extracellular Vesicles. Front Immunol 9:2349.

42. Hartmann S, Lucius R (2003) Modulation of host immune responses by nematode cystatins. Int J Parasitol 33: 1291-1302. [crossref]

43. Gregory WF, Maizels RM (2008) Cystatins from filarial parasites: evolution, adaptation and function in the host-parasite relationship. Int J Biochem Cell Biol 40: 1389-1398. [crossref]

44. Zavasnik-Bergant T (2008) Cystatin protease inhibitors and immune functions. Front Biosci 13:4625-37.

45. Coronado S, Barrios L, Zakzuk J, Regino R, Ahumada V, et al. (2017) A recombinant cystatin from Ascaris lumbricoides attenuates inflammation of DSSinduced colitis. Parasite Immunol 39. [crossref]

46. Janssen L, Silva Santos GL, Muller HS, Vieira AR, de Campos TA, de Paulo Martins V (2016) Schistosome-Derived Molecules as Modulating Actors of the Immune System and Promising Candidates to Treat Autoimmune and Inflammatory Diseases. J Immunol Res 2016: 5267485.

47. Danilowicz-Luebert E, Steinfelder S, Kuhl AA, Drozdenko G, Lucius R, Worm M, et al. (2013) A nematode immunomodulator suppresses grass pollen-specific allergic responses by controlling excessive Th2 inflammation. Int J Parasitol 43: 201-10.

48. Ziegler T, Rausch S, Steinfelder S, Klotz C, Hepworth MR, et al. (2015) A novel regulatory macrophage induced by a helminth molecule instructs IL-10 in CD4+ $\mathrm{T}$ cells and protects against mucosal inflammation. J Immunol 194: 1555-1564. [crossref]

49. Jang SW, Cho MK, Park MK, Kang SA, Na BK, Ahn SC, et al. (2011) Parasitic helminth cystatin inhibits DSS-induced intestinal inflammation via IL-10(+) F4/80(+) macrophage recruitment. Korean J Parasitol 49: 245-54.

50. Falcon CR, Masih D, Gatti G, Sanchez MC, Motran CC, Cervi L (2014) Fasciola hepatica Kunitz type molecule decreases dendritic cell activation and their ability to induce inflammatory responses. PLoS One 9: 114505.

51. Schnoeller C, Rausch S, Pillai S, Avagyan A, Wittig BM, et al. (2008) A helminth immunomodulator reduces allergic and inflammatory responses by induction of IL10-producing macrophages. J Immunol 180: 4265-4272. [crossref]

52. Whelan RA, Rausch S, Ebner F, Gunzel D, Richter JF, Hering NA, et al. (2014) A transgenic probiotic secreting a parasite immunomodulator for site-directed treatment of gut inflammation. Mol Ther 22: 1730-40.

53. Liu F, Cheng W, Pappoe F, Hu X, et al. (2016) Schistosoma japonicum cystatin attenuates murine collagen-induced arthritis. Parasitol Res 115: 3795-3806. [crossref]

54. Wang S, Xie Y, Yang X, Wang X, Yan K, Zhong Z, et al. (2016) Therapeutic potential of recombinant cystatin from Schistosoma japonicum in TNBS-induced experimental colitis of mice. Parasit Vectors 9:6.

55. Li H, Wang S, Zhan B, He W, Chu L, Qiu D, et al. (2017) Therapeutic effect of Schistosoma japonicum cystatin on bacterial sepsis in mice. Parasit Vectors 10: 222 .
56. Togre N, Bhoj P, Goswami K, Tarnekar A, Patil M, Shende M. (2018) Human filarial proteins attenuate chronic colitis in an experimental mouse model. Parasite Immunol 40.

57. Cho MK, Lee CH, Yu HS. (2011) Amelioration of intestinal colitis by macrophage migration inhibitory factor isolated from intestinal parasites through toll-like receptor 2. Parasite Immunol 33: 265-75.

58. Bendrat K, Al-Abed Y, Callaway DJ, Peng T, Calandra T, et al. (1997) Biochemical and mutational investigations of the enzymatic activity of macrophage migration inhibitory factor. Biochemistry 36: 15356-15362. [crossref]

59. Kleemann R, Kapurniotu A, Frank RW, Gessner A, Mischke R, et al. (1998) Disulfide analysis reveals a role for macrophage migration inhibitory factor (MIF) as thiol-protein oxidoreductase. $J \mathrm{Mol} \mathrm{Biol} \mathrm{280:} \mathrm{85-102.} \mathrm{[crossref]}$

60. Nguyen MT, Beck J, Lue H, Fünfzig H, Kleemann R, et al. (2003) A 16-residue peptide fragment of macrophage migration inhibitory factor, MIF-(50-65), exhibits redox activity and has MIF-like biological functions. J Biol Chem 278: 33654 33671. [crossref]

61. Zang X, Taylor P, Wang JM, Meyer DJ, Scott AL, et al. (2002) Homologues of human macrophage migration inhibitory factor from a parasitic nematode. Gene cloning, protein activity, and crystal structure. J Biol Chem 277: 44261-44267. [crossref]

62. Cho Y, Jones BF, Vermeire JJ, Leng L, DiFedele L, Harrison LM, et al. (2007) Structural and functional characterization of a secreted hookworm Macrophage Migration Inhibitory Factor (MIF) that interacts with the human MIF receptor CD74. J Biol Chem 282: 23447-56.

63. Bennuru S, Semnani R, Meng Z, Ribeiro JM, Veenstra TD, Nutman TB (2009) Brugia malayi excreted/secreted proteins at the host/parasite interface: stage- and gender-specific proteomic profiling. PLoS Negl Trop Dis. 3: 410.

64. Prieto-Lafuente L, Gregory WF, Allen JE, Maizels RM. (2009) MIF homologues from a filarial nematode parasite synergize with IL-4 to induce alternative activation of host macrophages. J Leukoc Biol 85: 844-54.

65. Mitre E, Taylor RT, Kubofcik J, Nutman TB (2004) Parasite antigen-driven basophils are a major source of IL-4 in human filarial infections. J Immunol 172: 2439-2445. [crossref]

66. Girgis NM, Gundra UM, Ward LN, Cabrera M, Frevert U, Loke P (2014) Ly6C(high) monocytes become alternatively activated macrophages in schistosome granulomas with help from CD4+ cells. PLoS Pathog 10:1004080.

67. Park SK, Cho MK, Park HK, Lee KH, Lee SJ, et al. (2009) Macrophage migration inhibitory factor homologs of anisakis simplex suppress $\mathrm{Th} 2$ response in allergic airway inflammation model via $\mathrm{CD} 4+\mathrm{CD} 25+$ Foxp $3+\mathrm{T}$ cell recruitment. $J$ Immunol 182: 6907-6914. [crossref]

68. Cho MK, Park MK, Kang SA, Park SK, Lyu JH, Kim DH, et al. (2015) TLR2dependent amelioration of allergic airway inflammation by parasitic nematode type II MIF in mice. Parasite Immunol 37: 180-91.

69. Kelley LA, Mezulis S, Yates CM, Wass MN, Sternberg MJ (2015) The Phyre2 web portal for protein modeling, prediction and analysis. Nat Protoc 10: 845-858. [crossref]

70. Pettersen EF, Goddard TD, Huang CC, Couch GS, Greenblatt DM, Meng EC, Ferrin TE (2004) UCSF Chimera - a visualization system for exploratory research and analysis. $J$ Comput Chem 25(13): 1605-12.

\section{Citation:}

Khatri V, Chauhan N, Kalyanasundaram R (2019) Filarial Parasite-Derived New Potential Bio-Therapeutic Agents For Inflammatory Bowel Diseases. Adv Gastroenterol Hepatol Endoscopy 1(1): 1-5. 